\title{
A Novel Cluster-Chain Channel Adaptive Routing Protocol in Wireless Sensor Networks
}

\author{
Xingcheng Liu \\ Dept. of Elec. and Comm. Engr. \\ Sun Yat-Sen University \\ Guangzhou, Guangdong, China \\ +86-20-84115909 \\ isslxc@mail.sysu.edu.cn
}

\author{
Xiaoxiang Bian \\ Dept. of Elec. and Comm. Engr. \\ Sun Yat-Sen University \\ Guangzhou, Guangdong, China \\ $+86-20-84113593$ \\ bxx1126@126.com
}

\author{
Haengrae Cho \\ Dept. of Computer Engr. \\ Yeungnam University \\ Gyungsan, Gyungbuk, Korea \\ +82-53-8102559 \\ hrcho@yu.ac.kr
}

\begin{abstract}
The energy constraint in wireless sensor networks is a crucial issue affecting the network lifetime and connectivity. To realize true energy saving in a wireless environment and ensure reliable communications, the noise condition of the wireless channel should be taken into account. In this paper, we propose a clusterchain routing protocol (CCRP). Besides, we devise an adaptive power adjustment strategy which can dynamically adjust transmission power according to the receiver noise condition and the distance between the transmitter and receiver to ensure the required Packet Reception Rate (PRR). The adaptive power adjustment strategy is incorporated into CCRP to form a novel protocol-cluster-chain channel adaptive routing protocol (CCARP). Simulation results indicate that CCRP and CCARP outperform LEACH by at least $300 \%$ for a $200 \mathrm{~m} \times 200 \mathrm{~m}$ network when the scenario of $1 \%$ dead nodes is considered.
\end{abstract}

\section{Categories and Subject Descriptors}

C.2.1 [Computer-communication networks]: Network Architecture and Design-Wireless communication.

\section{General Terms}

Algorithms, Performance, Reliability, Experimentation

\section{Keywords}

Adaptive routing protocol, power adjustment, cluster, wireless sensor network

\section{INTRODUCTION}

The advances in micro-electronic-mechanical systems (MEMS)[1] based sensor technology, coupled with low-power, low-cost digital signal processors (DSPs) and the rapid development in wireless technology have enabled wireless sensors to be deployed in large quantities to form wireless sensor networks (WSNs) for a wide variety of civilian and military applications [2]-[4]. Normally, a sensor node is usually powered by battery, and once

QShine 2008, July 28-31, 2008, Hong Kong, Hong Kong. Copyright 2008 ICST ISBN 978-963-9799-26-4

DOI 10.4108/ICST.QSHINE2008.3822 deployed, the sensor networks are unattended, and therefore battery replacement is impossible. As a result, the lifetime of the sensor networks depends heavily on the batteries. Communications among sensor nodes have been recognized as the major factor of energy dissipation in WSNs [5]. Consequently, many researchers have focused their research on developing energy efficient communication protocols for wireless sensor networks.

However, in dealing with energy efficient communication protocols, the noise condition of the wireless channels among the sensor nodes is ignored in most of the previously published literatures. The Low Energy Adaptive Clustering Hierarchy $(\mathrm{LEACH})$ protocol and its improvements are among the list [6][8]. These protocols all use the first order radio model that can adjust the transmission power only according to the distance between the transmitter and receiver. Neglecting the time-variant property of noise can lead to an unnecessary waste of precious battery resources and can result in rapid depletion of node energy. The idea that a channel adaptive protocol can result in significant savings in energy consumption motivates our study.

In this paper, we propose a cluster-chain routing protocol (CCRP). Then, to ensure reliable communications in wireless sensor networks and decrease energy consumption, we devise an adaptive power adjustment strategy. As the noise changes with time, the strategy can dynamically adjust the transmission power to make sure that the packet reception rate (PRR) is above the required level. Finally, we apply the adaptive power adjustment strategy into CCRP to form a novel protocol-cluster-chain channel adaptive routing protocol (CCARP).

The rest of this paper is organized as follows. In Section 2, some related works are introduced. Section 3 describes the novel CCRP and CCARP protocols. An adaptive power adjustment strategy is proposed in Section 4 while the simulation results are presented in Section 5. Finally, the conclusions are drawn in Section 6.

\section{RELATED WORKS}

To enable the scalability and energy efficiency in a sensor network composed of a large number of sensor nodes, a clusterbased hierarchy is an elegant solution [9]. LEACH [6] is a dominant representative protocol with clustering structure. LEACH uses randomized rotation of the cluster-heads to distribute the energy load among the sensor nodes in a network. However, there are still some limitations in LEACH. On the one hand, LEACH's random cluster-head selection is prone to leading 
a non-uniform distribution of the cluster-heads and thus increases the total energy dissipated in the network. On the other hand, the direct communications between the cluster-heads and the base station may consume much energy if the cluster-heads are far away from the base station.

S. Lindesy and C. S. Raghavendra [10] believed that further improvements could be obtained if each node communicated only with close neighbors, and only one designated node sent the aggregated data to the base station in each round. So they proposed the protocol-Power-Efficient Gathering in Sensor Information Systems (PEGASIS). The routing chain can be constructed by using a greedy algorithm [11]. However, the chain topology causes excessive delay. Besides, increasing distances between neighbors will have a significant effect on the performance of PEGASIS when the network area is expanded [12].

Energy-efficient Chain-cluster Routing protocol (ECR) [13] is proposed recently. In ECR, the network can be divided into several clusters with the same width. Nodes in the same cluster are organized to be a chain according to the values of the horizontal $\mathrm{x}$-coordinates at the coordinates from one side to the other. In each round of ECR, the cluster-head leader is selected by the base station according to the maximum remained-energy criterion among the sensor nodes. Then this cluster-head-leader is taken as the beginning node in the greedy algorithm. The cluster heads of neighbor clusters will be gradually generated with the greedy algorithm. Finally a cluster-head-chain is formed by these cluster-heads. However, the chain topology in each cluster introduces considerable delay.

To alleviate the disadvantages of LEACH, we propose the CCRP protocol, which takes advantage of both LEACH and PEGASIS. In the proposed CCRP, a more balanced cluster constructing method is proposed and an improved data transmission scheme between the cluster-heads and the base station is suggested. In certain applications, it is necessary to support reliability in sensor networks such that the successful probability of end-to-end transmission meets the required specifications. Thereafter, the novel CCARP protocol that can dynamically adjust the transmission power to ensure the required PRR is proposed.

\section{THE PROPOSED PROTOCOLS}

The operation of CCRP can be broken into rounds like LEACH. However, the network considered in CCRP is divided into several clusters by the base station. In each round, one cluster-head begins to be selected in each cluster, followed by going into a steady-state where the data are transferred to the base station.

\subsection{Cluster Constructing Phase}

LEACH adopts a random cluster-head selection algorithm [6], which ensures that none of the sensors are overloaded due to the added responsibility of being cluster-head. However, LEACH can not ensure that the cluster-heads are uniformly placed across the whole sensor network. As a result, the cluster-heads in LEACH may become concentrated in a certain region of the network, as shown in Fig. 1. In this case, nodes from the "cluster-head deprived" regions will dissipate a considerable amount of energy while transmitting their data to a faraway cluster-head. Furthermore, the number of cluster-heads is uncertain in each round because of the random cluster-head selection algorithm. These limitations motivate us to propose a novel cluster constructing method, which can ensure the uniform distribution of the cluster-heads in the network

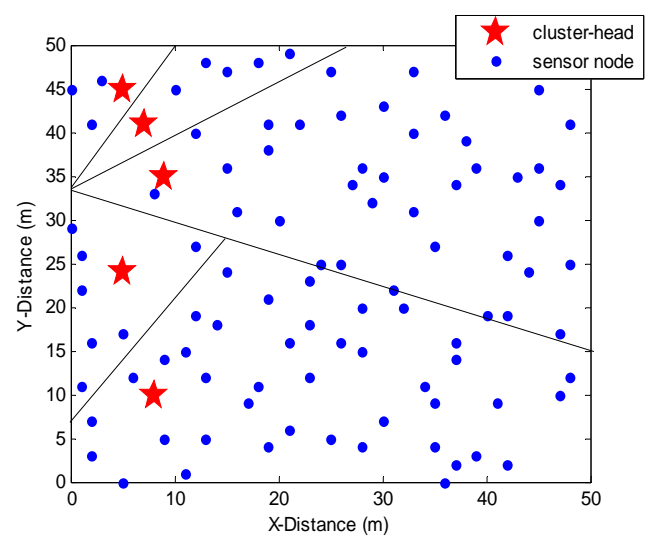

Fig. 1. Non-uniform distribution of cluster-heads in LEACH

In the proposed CCRP and CCARP, the clusters are constructed according to the $\mathrm{x}$-coordinates and $\mathrm{y}$-coordinates of sensor nodes. It is assumed that the base station knows the positional information of all other sensor nodes. Since the base station has strong processing capability and adequate energy, it can compute a cluster ID for all other nodes according to their positional information. Then the base station broadcasts a message containing a cluster ID to each node. Our cluster constructing method is different from the ECR employed by Y. Tian et al. [13]. The network in ECR is divided into several clusters only according to the distance in Y-direction between the sensed regions and the base station. Besides, the nodes in each cluster in the ECR scheme are organized into a chain, which introduces considerable delay when the network area is expanded and large enough. In our proposed CCRP and CCARP, the cluster-member nodes send data to the cluster-head directly. Furthermore, we propose an adaptive power adjustment strategy, which is not presented in literature [13]. So, the protocols proposed here and by Y. Tian et al. [13] are radically different.

In our study, the network topology is generated based on the random uniform distribution. A random 300-node network is shown in Fig. 2. The network in Fig. 2 can be divided into 16 clusters by the base station and the size of each cluster is $25 \mathrm{~m} \times 25 \mathrm{~m}$. The cluster constructing method can ensure the uniform distribution of the cluster-heads in the network and reduce the total energy dissipation of sensor nodes. 




Fig. 2. Network topology

\subsection{Cluster-head Selection Phase}

There are two phases in each round, which are the cluster-head selection phase and data transmission phase. One cluster-head must be selected in each cluster in each round. Generally speaking, the cluster-heads consume energy rapidly. The premature deaths of sensor nodes may cause the emergence of 'blind area' in the network coverage and decrease the quality of network surveillance [13]. Therefore, prolonging the time of the point at the node death ratio $1 \%$ is the primary aim to improve the network quality. The proposed CCRP includes a simple but efficient cluster-head selection algorithm.

While choosing which node to be acted as the cluster-head in a cluster, the remaining energy of the node and the distances between the node and the other nodes in the same cluster must be considered. At the beginning of each round, every node computes the priority of becoming the cluster-head $(P R I)$ :

$$
P R I_{i}=\frac{E_{c}}{\sum_{j=1}^{n} d_{i j}^{2}},
$$

where, $E_{c}$ is the current remaining energy of the node $i, d_{i j}$ is the distance between node $i$ and node $j$ in the same cluster with a total of $(n+1)$ nodes.

After the $P R I$ is computed, each node competes with each other to become the cluster-head. Each node broadcasts a very short message containing its cluster ID and PRI in the intra-cluster communication radius. The other node receives the message. If its cluster ID is identical with the cluster ID indicated in the message, it compares the received $P R I$ values with its own $P R I$ value. If its own $P R I$ value is the highest value, it will select itself as the cluster-head for the current round. If not, it will wait for a broadcast message from the other cluster-head. We ignore the energy consumed for transmitting and receiving the short message since the message is much smaller in length than the data packet.

Then the cluster-head broadcasts an advertisement message containing its cluster ID in its inter-cluster communication radius. The other non-cluster-head node receives this message and compares its cluster ID with the cluster ID in the message. If they are the same, the non-cluster-head node informs the cluster-head that it will be a member of the cluster for this round. The other cluster-heads report their positional information to the clusterhead after they receive the advertisement message.

The cluster-head node receives all the messages for nodes that are to be included in the cluster. Based on the number of nodes in the cluster, the cluster-head creates a TDMA schedule informing each node when it can transmit. Each sensor node is allowed to transmit packets in its own allocated time slots so that no collision occurs. The schedule is broadcast back to the nodes in the cluster. Particularly, in the proposed CCARP, the schedule message contains a field indicating the required received signal power so that the non-cluster-head node can adjust its transmission power using the adaptive power adjustment strategy introduced in Section 4 .

\subsection{Data Transmission Phase}

In LEACH, the cluster-heads communicate with the base station directly. For the cluster-heads that are far away from the base station, direct communications will consume much energy. Therefore, the proposed CCRP constructs a chain among the cluster-heads so that each cluster-head will receive from and transmit to an adjacent cluster-head.

It is supposed that there are $K$ cluster-heads in the inter-cluster communication radius of each cluster-head. Each cluster-head contains the following information which is required in constructing a chain:

1) Node ID-i;

2) Node position- $\mathrm{P}_{i}\left(\mathrm{X}_{i}, \mathrm{Y}_{i}\right)$

3) Distance between cluster-head $i$ and the base station- $-\mathrm{HB}_{i}$;

4) Distance set between cluster-head $i$ and the other cluster-heads-



The cluster-heads are joined to construct a chain with the following method.

1) The minimal value among distances between the cluster-heads and the base station is chosen. Suppose $\mathrm{HB}_{\mathrm{i}}$ is the minimal value, then the length of the chain is $L=\mathrm{HB}_{\mathrm{i}}$.

2) The minimal value from the set of distance between clusterhead $i$ and the other cluster-heads is chosen. Suppose cluster-head $x$ has the shortest distance to cluster-head $i$, i.e. $H_{i x}$ is the minimal value in the set $\mathrm{HH}_{i}$, then $L=\mathrm{HB}_{\mathrm{i}}+\mathrm{HH}_{i x}$.

3) The minimal value from the set of distance between clusterhead $x$ and the other cluster-heads is selected. Suppose $\mathrm{HH}_{\mathrm{xy}}$ is the selected minimal value in the set $\mathrm{HH}_{x}$, then $L=\mathrm{HB}_{\mathrm{i}}+\mathrm{HH}_{i x}+$ $\mathrm{HH}_{x y}$.

The above process continues until all the cluster-heads are included in the chain. Each cluster-head records the IDs of its prehop node and next-hop node.

Fig. 3 shows a snapshot of chain formation among the clusterheads using above method. The base station is located at $(50,200)$ in Fig. 3. 


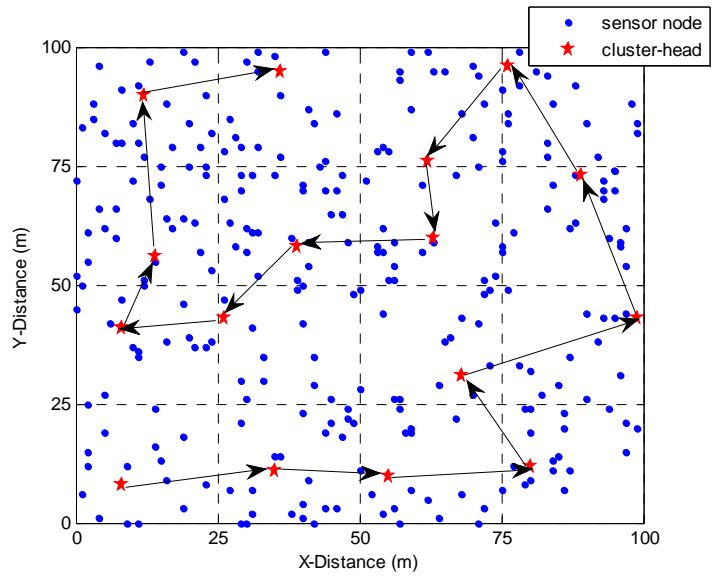

Fig. 3. A snapshot of chain formation

Once the TDMA schedule in each cluster is fixed and the chain is formed, data transmission can begin. It is assumed that all sensors are sensing the environment at a fixed rate and have data to send periodically. Each cluster-member node is allowed to transmit data to the cluster-head node during its own allocated time slot so that no collision can occur. When all the data have been received, the cluster-head node needs to aggregate the packets, which dissipates $5 \mathrm{~nJ} / \mathrm{bit} / \mathrm{message}[6]$. Here, the length of the aggregated packet is assumed to be the same as that of the original one. After all cluster-heads complete data gathering from their clustermember nodes, aggregated data are transmitted from one clusterhead to another along the chain. Eventually the aggregated data are delivered to the base station by the cluster-head that has the shortest distance to the base station.

\section{ADAPTIVE POWER ADJUSTMENT STRATEGY}

In the actual wireless communications, the wireless channel is time-variant. To realize true energy saving in wireless sensor networks, the time-variant property of the noise should be taken into account. It is of great significance to propose an adaptive power adjustment strategy that can exploit the time-variant nature of the noise and ensure the required PRR at the receiver side.

\subsection{Radio Model}

The proposed CCRP uses the first order radio model discussed in [6], which is given by:

$$
\begin{aligned}
& E_{T x}(k, d)=k E_{\text {elec }}+k d^{2} \varepsilon_{\text {amp }}, \\
& E_{R x}(k)=k E_{\text {elec }}
\end{aligned}
$$

where, $E_{\text {elec }}=50 \mathrm{~nJ} / \mathrm{bit}$ denotes the energy consumption of the electronic circuitry, $\varepsilon_{a m p}=100 \mathrm{pJ} / \mathrm{bit} / \mathrm{m}^{2}$ denotes the energy consumption of the transmitter amplifier.

In the proposed CCARP, the energy consumption for transmitting and receiving $k$ bits is given by [14]:

$$
\begin{aligned}
& E_{T x}(k)=k E_{\text {elec }}+k \frac{P_{t}}{R_{b}}, \\
& E_{R x}(k)=k E_{\text {elec }}
\end{aligned}
$$

where, $R_{b}$ is the data rate in bits per second, and $P_{t}$ is the transmission power.

\subsection{Packet Reception Rate}

In the proposed protocols, each non-cluster-head node is allowed to transmit packets at its own allocated time slots. Data packet aggregated by each cluster-head node is transmitted hop-by-hop along the chain. Since there is no interference from other nodes' transmissions, the transmission failure can occur only due to channel errors, which depend on the transmission power, channel gain, and receiver noise condition.

The bit error rate (BER) of non-coherent FSK (modulation scheme used in MICA2 motes) is given by [15]:

$$
p_{b}=\frac{1}{2} \exp \left[-\frac{1}{2} \frac{E_{b}}{N_{0}}\right],
$$

where, $p_{b}$ is the BER, $E_{b} / N_{0}$ is the ratio of energy per bit to the noise spectral density.

However, the $E_{b} / N_{0}$ metric is not provided in most cases, so the Signal-to-Noise Ratio (SNR) is adopted. Hence, the expression of BER vs $E_{b} / N_{0}$ can be converted to that of BER vs SNR.

The relation between $S N R$ and $E_{b} / N_{0}$ is given by:

$$
S N R=\frac{E_{b} R_{b}}{N_{0} B_{N}},
$$

where, $R_{b}$ is the data rate in bits per second, and $B_{N}$ is the system bandwidth. For MICA2 motes, $R_{b}=19.2 \mathrm{kbps}$ and $B_{N}=30 \mathrm{kHz}$. Finally, PRR can be calculated as follows [15]:

$$
p=\left(1-\frac{1}{2} \exp ^{-0.78125 S N R}\right)^{s}
$$

where, $p$ is the PRR, $s$ is the packet size in bits.

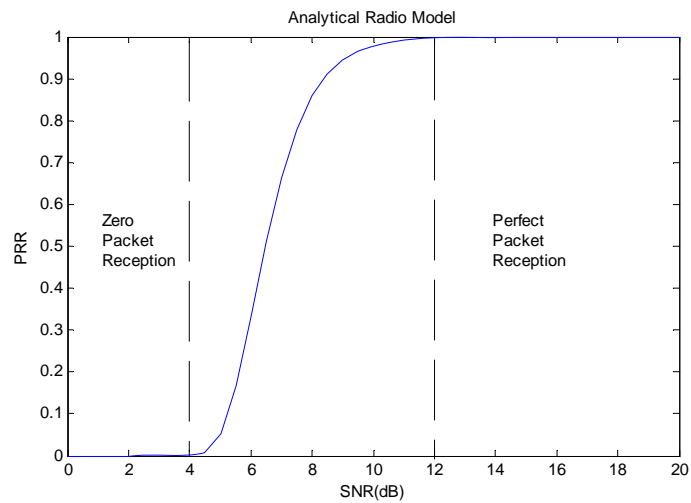

Fig. 4. Radio Model: Non-Coherent FSK, $s=100$ bytes

The curve in Fig. 4 demonstrates the scenario of Eq. (6) at different $S N R$ for a packet size of 100 bytes. As shown in Fig. 4, the packet reception rate is close to 1 when $S N R$ is above a threshold value of approximate $11 \mathrm{~dB}$.

ZigBee [16] is the global communications standard for developing compatible, reliable and low-power wireless solutions for residential and industrial applications. ZigBee devices are required to conform to the IEEE 802.15.4-2006 Wireless Personal 
Area Networks (WPANs) standard [17]. The PRR is required to be not less than $99 \%$ in the IEEE 802.15.4 standard. So, the required $S N R$ at the receiver side can be obtained according to Eq. (6), given by:

$S N R \geq 11.3 \mathrm{~dB}$.

\subsection{Adaptive Power Adjustment Strategy}

In our study, it is assumed that each sensor node can detect ambient environment noise level. The ambient environment noise is assumed to be an additive white Gaussian noise (AWGN), with mean 0 and variance $\sigma^{2}$.

Here, the SNR is defined as follows:

$S N R=P_{r} / P_{n}$,

where, $P_{r}$ is the received signal power, and $P_{n}$ is the noise power

Based on Eq. (6), the corresponding $S N R$ can be computed in each sensor node, given the required PRR. The noise power $P_{n}$ also can be obtained. Therefore, $P_{r}$ can be computed by Eq. (7).

In a wireless channel, the electromagnetic wave propagation meets the power-law function of the distance between the transmitter and the receiver. However, if the distance is less than a certain crossover distance, the Friis free space model is used (attenuation with $d^{2}$ ). Otherwise, the two-ray ground model is used (attenuation with $d^{4}$ ).

The crossover distance is defined as follows [18]:

$d_{\text {crossover }}=\frac{4 \pi \sqrt{L} h_{r} h_{t}}{\lambda}$,

where, $L \geq 1$ is the system loss factor, $h_{r}$ and $h_{t}$ are the heights of the receiver antenna and the transmitter antenna, and $\lambda$ is the wavelength of the carrier signal.

In this paper, it is assumed that an omni-directional antenna is used with the following parameters: antenna gain $G_{t}=G_{r}=1$, height $h_{t}=h_{r}=1.5 \mathrm{~m}$, loss factor $L=1$ (no system loss), and carrier frequency $915 \mathrm{MHz}$. Simple computations yield $d_{\text {crossover }}=86.2 \mathrm{~m}$.

Since the communication radius in our experiments is less than the crossover distance, the Friis free space model can be represented with Eq. (9).

$P_{t}=\frac{P_{r}(4 \pi d)^{2} L}{G_{t} G_{r} \lambda^{2}}$,

where, $P_{r}$ is the received power at the distance $d$ between the transmitter and the receiver, and $P_{t}$ is the transmission power.

Since the schedule message from the cluster-head contains a field indicating the required received signal power $P_{r}$ obtained from Eq. (7), the transmitter of the cluster-member node can adjust its transmission power $P_{t}$ according to Eq. (9). Since the proposed CCARP can ensure the required PRR performance, it is unnecessary for nodes to send ACK packets.

\section{SIMULATION AND ANALYSIS}

The performance of the proposed protocols is simulated with Visual $\mathrm{C}++$ 6.0. The adaptive power adjustment strategy is applied into CCRP to form CCARP. An AWGN noise is generated for each sensor node for simulating the noise of environment. Since the energy consumption has a close relation to the variance of noise, it is important to choose an appropriate $\sigma^{2}$. The average noise power is approximately $-105 \mathrm{dBm}$ [15]. Consequently, the value $10^{-13.5}$ is chosen as the variance. The other parameters used in simulations are shown in Table I.

\section{Table I Simulation parameters}

\begin{tabular}{|c|c|c|}
\hline \hline Parameters & Configuration 1 & Configuration 2 \\
\hline Network size $(\mathrm{m})$ & $(100 \times 100)$ & $(200 \times 200)$ \\
\hline Base station coordinates $(\mathrm{m})$ & $(50,200)$ & $(100,300)$ \\
\hline Node number & 300 & 1200 \\
\hline Cluster size(m) & \multicolumn{2}{|c|}{$25 \times 25$} \\
\hline Cluster-head probability & \multicolumn{2}{|c|}{0.05} \\
\hline $\begin{array}{c}\text { Intra-cluster communication } \\
\text { radius(m) }\end{array}$ & 35 \\
\hline $\begin{array}{c}\text { Inter-cluster communication } \\
\text { radius(m) }\end{array}$ & 70 \\
\hline Data packet size(bits) & \multicolumn{2}{|c|}{800} \\
\hline Broadcast packet size(bits) & 64 \\
\hline Schedule packet size(bits) & \multicolumn{2}{|c|}{64} \\
\hline Initial energy levels(J) & $0.25,0.5,1$ \\
\hline \hline
\end{tabular}

\subsection{Energy Consumption Analysis}

Table II summarizes the results of the network with the parameters of configuration 1 . As can be expected, the number of rounds doubles as the energy per node doubles for a given size of network.

Table II Number of rounds at different death proportions of nodes

\begin{tabular}{|c|c|c|c|c|c|}
\hline \hline $\begin{array}{c}\text { Energy } \\
\text { (J/node) }\end{array}$ & Protocol & $\begin{array}{c}\text { No. of } \\
\text { Rounds } \\
\mathbf{( 1 \% )}\end{array}$ & $\begin{array}{c}\text { No. of } \\
\text { Rounds } \\
\mathbf{( 2 0 \% )}\end{array}$ & $\begin{array}{c}\text { No. of } \\
\text { Rounds } \\
\mathbf{( 5 0 \% )}\end{array}$ & $\begin{array}{c}\text { No. of } \\
\text { Rounds } \\
\mathbf{( 1 0 0 \% )}\end{array}$ \\
\hline \multirow{3}{*}{0.25} & LEACH & 765 & 901 & 1093 & 1440 \\
\cline { 2 - 6 } & CCRP & $\mathbf{1 8 4 0}$ & $\mathbf{2 1 2 7}$ & $\mathbf{2 2 1 7}$ & $\mathbf{2 3 6 0}$ \\
\cline { 2 - 6 } & CCARP & $\mathbf{2 0 6 9}$ & $\mathbf{2 4 2 7}$ & $\mathbf{2 5 9 5}$ & $\mathbf{2 6 5 9}$ \\
\hline \multirow{3}{*}{0.5} & LEACH & 1540 & 1811 & 2208 & 2923 \\
\cline { 2 - 6 } & CCRP & $\mathbf{3 6 3 3}$ & $\mathbf{4 2 6 6}$ & $\mathbf{4 4 9 5}$ & $\mathbf{4 6 9 9}$ \\
\cline { 2 - 6 } & CCARP & $\mathbf{4 1 4 5}$ & $\mathbf{4 8 6 0}$ & $\mathbf{5 2 1 6}$ & $\mathbf{5 3 5 4}$ \\
\hline \multirow{3}{*}{1.0} & LEACH & 2991 & 3458 & 4213 & 5610 \\
\cline { 2 - 6 } & CCRP & $\mathbf{7 3 5 8}$ & $\mathbf{8 5 0 4}$ & $\mathbf{8 9 5 2}$ & $\mathbf{9 4 5 6}$ \\
\cline { 2 - 6 } & CCARP & $\mathbf{8 4 6 2}$ & $\mathbf{9 7 4 4}$ & $\mathbf{1 0 4 1 4}$ & $\mathbf{1 0 6 7 5}$ \\
\hline \hline
\end{tabular}

Fig. 5 shows the number of rounds until $1 \%, 20 \%, 50 \%$, and $100 \%$ of nodes die for a $100 \mathrm{~m} \times 100 \mathrm{~m}$ network when the initial energy value for each node is $0.5 \mathrm{~J}$. The proposed CCRP outperforms LEACH by about $136 \%, 136 \%, 104 \%$ and $61 \%$ when $1 \%, 20 \%, 50 \%$, and $100 \%$ of nodes die, respectively. The proposed CCARP outperforms LEACH by about $169 \%, 168 \%$, $136 \%$ and $83 \%$ when $1 \%, 20 \%, 50 \%$, and $100 \%$ of nodes die, respectively. 


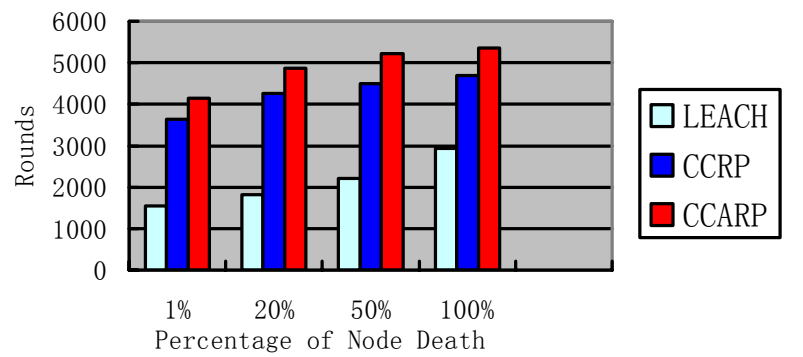

Fig. 5. Performance results with initial energy $0.5 \mathrm{~J} /$ node

Table III summarizes the results of the network with the parameters of configuration 2 .

Table III Number of rounds

at different death proportions of nodes

\begin{tabular}{|c|c|c|c|c|c|}
\hline \hline $\begin{array}{c}\text { Energy } \\
\text { (J/node) }\end{array}$ & Protocol & $\begin{array}{c}\text { No. of } \\
\text { Rounds } \\
\mathbf{( 1 \% )}\end{array}$ & $\begin{array}{c}\text { No. of } \\
\text { Rounds } \\
\mathbf{( 2 0 \% )}\end{array}$ & $\begin{array}{c}\text { No. of } \\
\text { Rounds } \\
\mathbf{( 5 0 \% )}\end{array}$ & $\begin{array}{c}\text { No. of } \\
\text { Rounds } \\
\mathbf{( 1 0 0 \% )}\end{array}$ \\
\hline \multirow{3}{*}{0.25} & LEACH & 425 & 561 & $\mathbf{7 7 4}$ & 1347 \\
\cline { 2 - 6 } & CCRP & $\mathbf{1 8 4 0}$ & $\mathbf{2 1 7 2}$ & $\mathbf{2 2 4 0}$ & $\mathbf{2 3 8 3}$ \\
\cline { 2 - 6 } & CCARP & $\mathbf{2 1 0 4}$ & $\mathbf{2 5 4 0}$ & $\mathbf{2 6 0 1}$ & $\mathbf{2 6 8 9}$ \\
\hline \multirow{3}{*}{0.5} & LEACH & 865 & 1131 & 1594 & 2813 \\
\cline { 2 - 6 } & CCRP & $\mathbf{3 7 7 0}$ & $\mathbf{4 3 5 6}$ & $\mathbf{4 4 8 0}$ & $\mathbf{4 7 9 4}$ \\
\cline { 2 - 6 } & CCARP & $\mathbf{4 3 6 2}$ & $\mathbf{5 1 0 4}$ & $\mathbf{5 2 2 8}$ & $\mathbf{5 3 5 5}$ \\
\hline \multirow{3}{*}{1.0} & LEACH & 1714 & 2273 & 3149 & 5565 \\
\cline { 2 - 6 } & CCRP & $\mathbf{7 3 2 8}$ & $\mathbf{8 7 9 3}$ & $\mathbf{9 0 2 8}$ & $\mathbf{9 5 9 4}$ \\
\cline { 2 - 6 } & CCARP & $\mathbf{8 3 1 5}$ & $\mathbf{1 0 2 1 9}$ & $\mathbf{1 0 4 8 3}$ & $\mathbf{1 0 7 2 4}$ \\
\hline \hline
\end{tabular}

When the initial energy value for each node is $0.5 \mathrm{~J}$, the proposed CCRP outperforms LEACH by about $336 \%, 285 \%, 181 \%$ and $70 \%$ when $1 \%, 20 \%, 50 \%$, and $100 \%$ of nodes die, respectively. The proposed CCARP outperforms LEACH by about $404 \%$, $351 \%, 228 \%$ and $90 \%$ when $1 \%, 20 \%, 50 \%$, and $100 \%$ of nodes die, respectively.

If we define the lifetime of the sensor network as the time between the network beginning and $1 \%$ node death, we can conclude that the proposed CCRP and CCARP could effectively improve the network lifetime compared to LEACH, especially when the network size is enlarged according to the results shown in Table II and III. When the network size is $100 \mathrm{~m} \times 100 \mathrm{~m}$, the lifetimes of the proposed CCRP and CCARP are about 1.5 times longer than LEACH. When the network size is $200 \mathrm{~m} \times 200 \mathrm{~m}$, the lifetimes of the proposed CCRP and CCARP are about 3.5 times longer than LEACH.

The better performance is obtained due to several reasons. The novel cluster constructing method and cluster-head selection algorithm can obtain a uniform distribution of the cluster-heads in the network, which can decrease the communication distance between the cluster-heads and their members and reduce the energy consumption. The dynamic cluster-head selection distributes the energy consumption among the nodes in the network. The multi-hop communications between the clusterheads and the base station are effective in reducing energy dissipation when the network size is large. In the proposed CCARP, each sensor node using the adaptive power adjustment strategy can adapt to the dynamic wireless channels in order to minimize energy consumption.

\subsection{Delay Analysis}

In LEACH, $5 \%$ of the total nodes act as cluster-heads. Thus, for a 300-node network there are about 15 long-distance transmissions from 15 cluster-heads to the base station. In addition, LEACH utilizes the TDMA schedule to gather information from the cluster-member nodes to the cluster-head. Delay time in one round can be estimated as the following: there are approximately 20 nodes per cluster for a 300-node network. If $t$ unit of time is required for one node to transmit data to the cluster-head, the cluster-head requires about $19 t$ units of time to collect data from cluster-member nodes. Then the 15 cluster-heads need extra $15 t$ units of time to transmit data to the base station. In total the time delay is $34 t$.

In case of PEGASIS, all the nodes in the network form a chain using greedy algorithm. During each round, the nodes among the chain take turns to collect and transmit data to the base station. So the number of long-distance transmissions reduces to minimum but introduces an excessive delay. Here the unit time delay $t$ to transmit from one node to the next node is assumed to be the same. For a 300-node network, if the leader is the end node in the chain, the other end node needs $299 t$ units of time to reach the leader. The leader needs extra $t$ units of time to transmit the data to the base station. So the delay can be $300 t$ units, which is considerably high.

In case of the proposed CCRP and CCARP, the network is divided into several clusters by the base station. For a 300-node network which is divided into 16 clusters, there are approximately 19 nodes per cluster. If $t$ unit of time is required for one node to transmit data to the cluster-head, the cluster-head requires about $18 t$ units of time to collect data from cluster-member nodes. Then the 16 cluster-heads are formed into a chain using greedy algorithm. The chain-leader is cluster-head which is the nearest to the base station. The other end cluster-head needs $15 t$ units of time to reach the leader and $t$ unit of time to reach the base station. So for a 300-node network, the delay can be $34 t$ units. Therefore, the proposed CCRP and CCARP give a good compromise between energy efficiency and delay.

\subsection{Reliability Analysis}

Many routing protocols in WSNs are evaluated through simulations, where simple assumptions about the link layer were made, such as the idealized perfect-reception-within-range model [19]. However, it is unrealistic in the actual wireless communications. In our proposed CCARP, the time-variant property of the wireless channel is taken into account. It can adjust the transmission power to ensure the required PRR performance at the receiver side. Therefore, the proposed CCARP is of importance in the applications that require high reliability of data transmission.

\section{CONCLUSIONS}

In this paper, we proposed a novel CCRP protocol, which adopts a cluster constructing method that can obtain a uniform distribution of the cluster-heads in the network. Furthermore, the proposed CCRP improves the data transmission mechanism from the cluster-heads to the base station via constructing a chain among the cluster-heads. Since the proposed protocol, CCRP, evenly distributes energy consumption among the sensor nodes in 
the network, the network lifetime can be prolonged. Simulations show that, for a $200 \mathrm{~m} \times 200 \mathrm{~m}$ network with the initial energy $0.5 \mathrm{~J}$ in each node, the proposed CCRP outperforms LEACH by about $336 \%, 285 \%, 181 \%$ and $70 \%$ when $1 \%, 20 \%, 50 \%$, and $100 \%$ of nodes die, respectively.

In addition, to ensure reliable communications in wireless sensor networks and decrease energy consumption, we devised an adaptive power adjustment strategy, where the transmitters of sensor nodes can adapt to the dynamic wireless channels in order to minimize energy consumption while ensuring the required PRR performance. This strategy is incorporated into CCRP to form a novel CCARP. Simulations show that the CCARP outperforms LEACH by $404 \%, 351 \%, 228 \%$ and $90 \%$ when $1 \%, 20 \%, 50 \%$, and $100 \%$ of nodes die, respectively, for a network of area $200 \mathrm{~m} \times 200 \mathrm{~m}$ with the initial energy $0.5 \mathrm{~J}$ in each node.

\section{ACKNOWLEDGMENTS}

This research was supported by the National Natural Science Foundation of China (No. 60673086, 607111 40419), the Science and Technology Plan of Guangdong Province of China (No. 2006B50101003), and the Joint KOSEF Project (F01-2007-00010062-0)

\section{REFERENCES}

[1] A. Chandrakasan, R. Amirtharajah, S. Cho, et al, "Design Considerations for Distributed Microsensor Systems," in Proc. IEEE Custom Integrated Circuits Conference (CICC), Piscataway, NJ, USA, pp. 279-286, May 1999.

[2] I. F. Akyildiz, W. Su, Y. Sankarasubramaniam, et al, "A Survey on Sensor Networks," IEEE Communications Magazine, 40(8): 102-114, Aug. 2002.

[3] P. Bonnet, J. Gehrke, P. Seshadri, "Querying the Physical World," IEEE Personal communications, 7(5):10-15, Oct. 2000 .

[4] C. Y. Chong, S. P. Kumar, "Sensor Networks: Evolution, Opportunities, and Challenges," in Proc. IEEE, 91(8): 12471256, Aug. 2003.

[5] V. Raghunathan, C. Schurgers, S. Park, et al, "Energy-aware Wireless Microsensor Networks," IEEE Design \& Test of Computers, 18(2): 62-74, 2001.

[6] W. R. Heinzelman, A. Chandrakasan, H. Balakrishnan, "Energy-Efficient Communication Protocol for Wireless Microsensor Networks," in Proc. the 33rd Annual Hawaii International Conference on System Sciences, Hawaii, USA, Vol. 8, pp. 3005-3014, Jan. 2000.

[7] M. J. Handy, M. Haase, D. Timmermann, "Low Energy Adaptive Clustering Hierarchy with Deterministic Cluster-
Head Selection," in Proc. the 4th IEEE Conference on Mobile and Wireless Communications Networks, Stockholm, Sweden, pp. 368-372, Sept. 2002.

[8] R. S. Chang, C. J. Kuo, "An Energy Efficient Routing Mechanism for Wireless Sensor Networks," in Proc. the 20th IEEE Conference on Advanced Information Networking and Applications, Vienna, Austria, Vol. 2, pp. 18-20, Apr. 2006.

[9] G. Huang, X. Li, "Energy-Efficiency Analysis of ClusterBased Routing Protocols in Wireless Sensor Networks," IEEE Aerospace Conference, Big Sky, Montana, USA, pp. 1-8, Mar. 2006.

[10] S. Lindesy, C. S. Raghavendra, "PEGASIS: Power-Efficient Gathering in Sensor Information System," in Proc. IEEE Aerospace Conference, Big Sky, Montana, USA, pp. 1-6, Mar. 2002.

[11] P. E. Black, greedy algorithm, available: http://www.nist. gov/dads/HTML/greedyalgo.html, Feb. 2005.

[12] S. D. Muruganathan, D. C. F Ma, R. I. Bhasin, et al, "A Centralized Energy-Efficient Routing Protocol for Wireless Sensor Networks," IEEE Radio Communications, 43(3): 813, Mar. 2005.

[13] Y. Tian, Y. Wang, Shu-Fang Zhang, "A Novel Chain-Cluster Based Routing Protocol for Wireless Sensor Networks," in Proc. Wireless Communications, Networking and Mobile Computing, Shanghai, P.R. China, pp. 2456-2459, Sep. 2007.

[14] Y. Yuan, Z. Yang, J. He, et al, "An Adaptive Code Position Modulation Scheme for Wireless Sensor Networks," IEEE communications letters, 9(6): 481-483, Jun. 2005.

[15] M. Zuniga, B. Krishnamachari, "Analyzing the Transitional Region in Low Power Wireless Links," in Proc. IEEE Sensor and Ad Hoc Communications and Networks (SECON), Santa Clara, Canada, Vol. 1, pp. 517-526, Oct. 2004.

[16] ZigBee Alliance, available: http://www.zigbee.org/en/ index.asp, 2007.

[17] GetIEEE802 download, available: http://standards.ieee.org/ getieee802/download/802.15.4-2006.pdf, 2006.

[18] W. B. Heinzelman, "Application-Specific Protocol Architectures for Wireless Networks," PhD. dissertation, Dept. of Electrical Engineering and Computer Science, MIT, Jun. 2000

[19] A. Woo, T. Tong, D. Culler, "Taming the Underlying Challenges of Reliable Multihop Routing in Sensor Networks," in Proc. the ACM Conference on Embedded Networked Sensor Systems, Los Angeles, CA, pp. 14-27, Nov. 2003. 